\title{
Apports et limites du numérique dans la transcription des manuscrits de Madame Bovary
}

Danielle Girard

\section{(2) OpenEdition}

\section{Journals}

Édition électronique

URL : http://journals.openedition.org/recherchestravaux/113

DOI : 10.4000/recherchestravaux. 113

ISSN : 1969-6434

Éditeur

UGA Éditions/Université Grenoble Alpes

\section{Édition imprimée}

Date de publication : 15 juin 2008

Pagination : 241-248

ISBN : 978-2-84310-125-0

ISSN : 0151-1874

Référence électronique

Danielle Girard, « Apports et limites du numérique dans la transcription des manuscrits de Madame Bovary », Recherches \& Travaux [En ligne], 72 | 2008, mis en ligne le 15 décembre 2009, consulté le 03 février 2021. URL : http://journals.openedition.org/recherchestravaux/113 ; DOI : https://doi.org/ 10.4000/recherchestravaux.113 


\section{Apports et limites du numérique dans la transcription des manuscrits de Madame Bovary ${ }^{1}$}

Les manuscrits de Madame Bovary ont été confiés en I 9 I 4 à la Bibliothèque municipale de Rouen² par la nièce de Gustave Flaubert. En 2003, la bibliothèque les a numérisés en images de très haute définition et le Centre Flaubert de l'Université de Rouen'3 a assuré l'organisation de leur transcription.

L'ensemble est consultable sur Internet dans deux sites conçus pour répondre aux différentes attentes des visiteurs. Sur le site Bovary ${ }^{4}$ se trouvent les manuscrits avec leur transcription, qu'on peut afficher séparément ou simultanément dans plusieurs modes de navigation : selon l'ordre de la narration, selon le classement génétique des manuscrits, ou dans l'ordre matériel de foliotage. On pourra aussi feuilleter les illustrations de deux éditions de Madame Bovary.

L'Atelier Bovary' ne contient pas les images des manuscrits, mais regroupe tous les outils nécessaires à la recherche et des dossiers plus «littéraires» sur les normandismes, les voix narratives, les notes de régie, l'index des noms propres, la cartographie. Son ergonomie simplifiée le destine plutôt aux étudiants en lettres et aux lycéens.

I. Une version allégée de cet article a été publiée par D. Girard dans la revue des Études Normandes, sous le titre «Numériser Flaubert», Mont-Saint-Aignan, 2007, n 4, p. 9-I6.

2. La Bibliothèque municipale de Rouen : http://bibliotheque.rouen.fr.

3. Le Centre Flaubert (CÉRÉdI) : http://flaubert.univ-rouen.fr.

4. Un problème informatique retarde quelque peu l'ouverture de ce site à l'adresse : http://www.bovary.fr.

5. Adresse de L'Atelier Bovary : http://flaubert.univ-rouen.fr/bovary/atelier/atelier.php. 


\section{L'appel à transcripteurs}

L'écriture de Flaubert ne serait pas très difficile à lire s'il ne s'acharnait sur son texte. «Il travaille avec une obstination féroce, écrit, rature, recommence, surcharge les lignes, emplit les marges, trace des mots en travers, et sous la fatigue de son cerveau il geint comme un scieur de long», écrit Maupassant dans un article paru dans Le Gaulois, le 23 août I 880. On peut avoir ainsi une dizaine de folios reprenant les mêmes expressions jusqu'à l'état final. Les rencontres d'Emma et Rodolphe tout au début de leur liaison (II, chap. 9) sont travaillées sur 52 folios pour aboutir à 38 lignes dans le texte édité6.

La difficulté et l'ampleur de la tâche ${ }^{7}$ - il y avait 4546 folios à transcrire ont nécessité des appels au bénévolat. Cent trente transcripteurs y ont répondu. Si l'on ajoute les élèves des 17 classes de seconde travaillant sous la direction de leur professeur, on peut estimer à 600 le nombre de ceux qui ont participé au projet.

Ils ont de I 6 à 76 ans et habitent en France métropolitaine, mais aussi en Argentine, Autriche, Belgique, Colombie, Ghana, Grande-Bretagne, Hongrie, Italie, Japon, Nouvelle-Calédonie, Nouvelle-Zélande, Polynésie française, Portugal, Suisse, Thailande, USA.

Leurs activités sont très diverses : chercheurs spécialistes de Flaubert, étudiants, lycéens, radiologue, prospecteur de pétrole, historien d'art, professeur de physique, bibliothécaire, psychiatre, éditeur, libraire, femme de ménage, traducteur, documentaliste dans un service de travailleurs sociaux, musicologue, etc.

\section{L'organisation du travail}

Nous avons réparti les documents de manière à ce que chacun ait à transcrire une vingtaine de folios, aboutissant tous à une ou deux pages du manuscrit définitif. La liste des séquences ainsi constituées a été affichée sur le site Flaubert de l'Université de Rouen. Les «candidats» ont pu en choisir librement une ou plusieurs, selon leur goût et le temps dont ils disposaient.

Leur tâche consistait à déchiffrer les textes et à les saisir en Word selon les codes que nous avons établis : des codes simples à appliquer lors de la saisie, simples à comprendre pour les publics de tous horizons qui liront les transcriptions. Nous avons donc opté pour une édition «analogique» qui tente

6. Voir p. 292 de l'édition électronique, ou page I 67 de l'édition de Cl. Gothot-Mersch, Garnier, I97 I.

7. L'indexation des transcriptions recense plus de 2500000 mots. 
de reproduire au mieux la disposition de la page en utilisant les fonctions de base de Word et de l'HTML et qui restitue bien tout ce qui indique la progression de l'écriture de Flaubert.

Les transcriptions achevées ont été mises en ligne immédiatement. Cela a créé une dynamique : voyant que leur travail était tout de suite consultable et exploitable, beaucoup ont repris une séquence. Entre le premier appel lancé le I6 mars 2004 et le dernier folio reçu - le is septembre 2006 - il ne s'est écoulé que deux ans et demi.

Pour parvenir au résultat le plus parfait possible, en même temps que le CD-ROM contenant les images de bonne définition ( 300 dpi) et un engagement sur les conditions d'utilisation, les transcripteurs ont reçu des consignes pour la saisie du texte, des indications pour afficher simultanément le traitement de texte et le folio à déchiffrer sur deux fenêtres ou deux écrans, quelques conseils sur la manière de procéder ainsi que la page du tableau génétique concernant la séquence à transcrire.

Dans le site Internet, la navigation repose entièrement sur ce classement génétique, établi par Marie Durel en 2000. Grâce à l'interface de Jean-Eudes Trouslard, en cliquant sur les barres qui représentent les brouillons très exactement situés par rapport au manuscrit définitif de Flaubert, on voit s'afficher l'image et la transcription du manuscrit.

Ces tableaux ont souvent permis de déchiffrer des mots ou des passages illisibles qu'on a pu restituer en se référant à un autre état du même paragraphe. Cela a surtout donné du sens et un éclairage au travail écrasant et souvent fastidieux de la transcription. Grâce à ce classement, il était possible de ne pas travailler à l'aveuglette : on avait tout de suite le plaisir intellectuel de suivre les affres de Flaubert, ses trouvailles, ses repentirs.

\section{Les conséquences du recours au bénévolat}

Le recours à la réalisation collective, outre l'organisation qu'elle a nécessité, a eu deux conséquences :

I. L'encodage des folios

L'une est purement technique et découle précisément de la multiplicité des intervenants. Sous un affichage uniforme en apparence, les fichiers - côté code - sont très différents. Selon les logiciels utilisés, la maittrise des scripteurs en traitement de texte, le pays d'origine, etc., on peut arriver à I I 24 lignes de code pour un folio original qui n'en compte que 50 !

Nous avons heureusement pu y remédier grâce à une «moulinette», conçue par l'informaticien Jean-Eudes Trouslard, qui supprime les innombrables balises de Microsoft. 
Ainsi débarrassés de toutes leurs «scories», les folios sont devenus des fichiers HTML parfaitement normalisés et pourront ultérieurement être encodés en XML.

2. La validité scientifique des transcriptions

L'autre question qui se pose, c'est la validité scientifique de ces documents établis par des «non-spécialistes». Notons d'abord qu'on ne naît pas spécialiste, on le devient. Notons aussi que l'enthousiasme et la passion n'empêchent en rien l'exigence de rigueur.

Nous nous sommes appuyés sur les travaux scientifiques antérieurs : le classement génétique de Marie Durel ${ }^{8}$, la transcription des comices agricoles par Jeanne Goldin ${ }^{9}$ en 1984 ou de la soirée à l'opéra par Matthew MacNamara ${ }^{10}$ en 2003.

Les transcripteurs n'ont pas été laissés seuls face à leurs problèmes. Nous leur avons donné des consignes précises et signalé les outils de référence présents sur le net.

La mise en service du moteur de recherche, au cours des relectures, a permis de s'assurer, quand un mot paraissait incertain, que Flaubert l'employait bien ailleurs dans Madame Bovary, et avec la même acception.

Un dialogue s'est instauré; il y a eu plusieurs milliers de messages échangés pour proposer une lecture de certains mots illisibles ou corriger des erreurs. Des chercheurs renommés pour leurs études flaubertiennes se sont chargés de quelques séquences ou nous ont écrit pour signaler des erreurs de lecture en nous montrant comment corriger.

Les transcriptions ont été vérifiées par six transcripteurs chevronnés qui ont acquis au fil du temps une parfaite connaissance de l'écriture de Flaubert entre i 85 i et i 856 .

Nous avons conscience que sur les centaines de milliers de mots qui composent le corpus, il y aura bien des retouches à faire! Mais cette édition électronique n'est pas figée. Nous la considérons comme un chantier permanent qui se modifiera encore selon les trouvailles des lecteurs.

8. M. Durel, «Classement et analyse des brouillons de Madame Bovary de Gustave Flaubert», thèse de Doctorat, Université de Rouen, 2000.

9. G. Flaubert, Madame Bovary (Extrait). Les Comices agricoles de Gustave Flaubert; transcription intégrale et genèse dans le manuscrit $G 223$ par J. Goldin, Genève, Droz, I 984.

I o. M. MacNamara, La Textualisation de Madame Bovary, Amsterdam et New York, Rodopi, 2003. 


\section{L'aide d'Internet dans le déchiffrement des manuscrits}

I. Dictionnaires en ligne et sites spécialisés

Pour vérifier l'existence d'un mot peu lisible, comprendre le sens d'une expression vieillie, repérer un normandisme, les dictionnaires sont des auxiliaires précieux. Internet en propose des centaines, des plus généralistes aux plus spécialisés ${ }^{11}$.

Au premier rang de tous, le Trésor de la langue française $e^{12}$ permet des contrôles rigoureux. Grâce à ses nombreuses citations, il valide parfois certaines hypothèses de lecture en montrant que Flaubert utilisait tel ou tel mot, dans la même acception.

Les noms propres sont difficiles à déchiffrer avec certitude.

Pour les toponymes, on peut interroger les sites consacrés à la généalogie ${ }^{13}$ qui répertorient les communes et les paroisses dont certaines sont aujourd'hui disparues, ou consulter les cartes extrêmement précises de Cassini ${ }^{14}$. Pour distinguer les lieux réels des lieux fictifs, les sites qui calculent les itinéraires sont utiles : on y vérifie par exemple que Belmesnil est bien un lieu que Charles peut atteindre depuis Tostes lors de ses consultations, mais que Barneville «près d'Aumale» est un lieu fictif.

2. La recherche libre sur Google

Le plus agaçant pour le transcripteur, ce sont les mots bien écrits, à peu près lisibles mais dont on ne saisit ni le sens, ni la raison d'être dans le manuscrit. Pourquoi «ta cavale isabelle» en marge d'un après-midi d'amour à Rouen? Pourquoi «battre l'eau»? Que fait Homais «en riochant»? Une recherche de ces expressions dans Google conduit à explorer plusieurs dictionnaires et sites dédiés à une œuvre ou un auteur. On découvre ainsi une allusion à un poème de Musset ${ }^{15}$, ou à la dure condition des serfs au Moyen Age consignée par tel ou tel dictionnaire encyclopédique du XIX siècle $^{16}$, ou encore l'usage d'un de

I I. Citons par exemple ClicNet Dictionnaires, http://clicnet.swarthmore.edu/dictionnaires.html.

I 2. Le Trésor de la Langue Française informatisé (TLFi) : http://atilf.atilf.fr/tlf.htm.

I 3. Communes de Seine-Maritime : http://lpessiot.free.fr/normandiegw/76/communes.htm.

I4. Les cartes de Cassini : http://www.cadeaux.com/v2/services_cassini/produit_cassini_choixi.asp.

I 5 . A. de Musset, «Le Lever», vers 2, Premières poésies I 829-I 835 , éd. par Maurice Allem :

«Assez dormir, ma belle! / Ta cavale isabelle / Hennit sous les balcons.

I6. Google repère cette expression, «battre l'eau», dans le Dictionnaire encyclopédique de Lebas (droits seigneuriaux). Almanach bistorique et statistique de l'Yonne, édition de l'année i 863. 
ces normandismes presque toujours supprimés par Flaubert ${ }^{17}$. On y trouve aussi parfois l'image des objets cités, telles ces statuettes en réduction de la Vénus de Médicis et de l'Apollon du Belvédère dont on comprend mieux alors la présence dans la vitrine du coiffeur chez qui Emma va faire lisser ses bandeaux au sortir de l'hôtel de Bourgogne.

Pour ce qui est des noms d'auteurs et d'œuvres, il y a presque toujours dans les profondeurs de Google l'article d'un érudit, spécialiste d'AbrahamHyacinthe Anquetil ou de «l'illustre Cadet de Gassicourt ${ }^{18}$ », qui vient confirmer la justesse du déchiffrement... et la malice de Flaubert qui met en lumière, avec ces auteurs de seconde zone, l'inculture de Charles ou le pédantisme d'Homais.

\section{Les apports et les limites de l'informatique}

\section{L'édition du texte en ligne}

Pour établir une édition de Madame Bovary qui soit le plus rigoureuse possible, nous avons utilisé la fonction de Word qui compare les états différents d'un même texte.

En croisant trois éditions déjà présentes sur le Internet, en nous reportant pour chaque différence signalée à l'édition Charpentier de I 873, nous avons fait plus de 400 corrections et produit un texte dont nous savons bien qu'il n'est sans doute pas irréprochable mais qu'il pourra encore être amendé.

2. Les transcriptions «analogiques»

Les fichiers HTML n'ayant pas la longueur prédéterminée d'une feuille de papier, nous avons pu choisir une disposition du texte très proche des feuillets de brouillons, en laissant à leur place les ajouts interlinéaires. Une impression des manuscrits de Madame Bovary tels que nous les avons transcrits comprendrait au moins 7000 pages, d'un texte qu'on ne pourrait plus modifier. On imagine mal un éditeur acceptant de se lancer dans une telle aventure! Les généticiens contournent habituellement l'obstacle en ayant recours à des transcriptions linéaires qui nécessitent des codes particuliers et rendent parfois la lecture moins évidente et accessible seulement à la communauté des chercheurs.

I7. Si l'on cherche «riocher» dans le moteur de recherche de Google, la première réponse renvoie à l'article «Rioter» du Littré (rire dédaigneusement) où, effectivement, la forme «riocher» est signalée comme un normandisme.

I 8. Anquetil (173I-I805) est, avec l'abbé Barthélemy (I716-I795), le seul auteur lu par Charles au collège. Cadet de Gassicourt est un «savant des bords du Rhin» que cite Homais pour se mettre en valeur devant le docteur Larivière. 
3. L'apport des transcriptions numérisées

Les transcriptions ont pour fonction première de guider et d'éclairer la lecture des manuscrits. Mais la saisie informatique a eu d'importantes répercussions, notamment la possibilité d'interroger la base de données avec un moteur de recherche spécialement créé à cet effet. Grâce à lui, on peut faire apparaitre les mots dans leur contexte et exporter les résultats pour mener à loisir une étude plus approfondie.

S'est posé alors le problème des noms propres. Les brouillons de Madame Bovary abondent en références culturelles. Comment savoir quels auteurs, quels personnages y sont cités et peuvent donc faire l'objet d'une requête? Une relecture sélective des folios a permis d'en faire le relevé et d'établir des index.

L'index des toponymes a servi à son tour à dresser la cartographie de Rouen et de la Seine Inférieure d'après des cartes datant de la Monarchie de Juillet.

4. Les limites du moteur de recherche

Le moteur de recherche a été difficile à concevoir pour des raisons qui tiennent à la spécificité du corpus :

$\mathrm{I}^{\circ} \mathrm{Il}$ ne s'agit pas d'un long roman en continu, mais de milliers de fragments.

$2^{\circ}$ Pour mettre à leur place les ajouts interlinéaires ou marginaux, nous avons dû disposer le texte dans des cellules de tableau.

$3^{\circ}$ Les pages recto et verso d'un même folio appartiennent à des passages différents - Flaubert réutilisait le papier usagé - ce qui complique considérablement l'ordre d'affichage des résultats de requête.

$4^{\circ}$ D'un brouillon à l'autre, Flaubert reprend constamment les mêmes phrases. Chaque mot un peu signifiant a des centaines, voire des milliers d'occurrences dans les brouillons.

Nous avons souhaité mettre en ligne un site gratuit, ouvert à tous, facile à utiliser même quand on ne dispose pas du haut débit ou de l'ordinateur le plus moderne et le plus performant. Nous craignons toutefois une grande lenteur dans le fonctionnement du moteur de recherche, notamment dans l'affichage des mots dans leur contexte.

\section{Les perspectives}

L'apport de l'informatique a été décisif à toutes les étapes de la mise en œuvre du projet. Un monde sépare l'armoire où Caroline rangeait les brouillons de son oncle Gustave et le site où l'on ira les consulter. Grâce à 
cette édition électronique, les manuscrits ne seront plus un objet inamovible, fragile, sacré. On les lira à volonté depuis tous les pays du monde, dans l'ordre qu'on choisira, sans autorisation préalable, sans se déplacer à Rouen, sans enfiler des gants blancs pour en tourner les pages.

Les enrichissements qu'on pourra y apporter sont multiples. La sagesse sera surtout de savoir s'imposer des limites. Nous voudrions que le site Bovary soit un simple tremplin pour toutes les recherches à venir. On n’y trouvera «que» les documents bruts : l'image des manuscrits avec l'écriture si attachante de Flaubert, les transcriptions, les index, les notes de régie, les cartes, les illustrations de quelques éditions du roman, avec un moteur de recherche qui permettra d'interroger la base de données. À partir de là, chacun selon son niveau, selon son projet, entreprendra des recherches personnelles.

Nous serions heureux si cette réalisation collective inédite pouvait donner à ceux qui hésitent le courage de se lancer dans une expérience similaire. Qu'ils profitent de nos erreurs, de nos tâtonnements. Qu'ils nous écrivent s'ils pensent qu'on peut les aider; nous le ferons de grand cœur ${ }^{19}$.

I 9. On trouvera à l'adresse suivante une version électronique de cet article, illustrée par des extraits des manuscrits de Flaubert et une démonstration des outils de recherche mentionnés plus haut: http://flaubert.univ-rouen.fr/bovary/atelier/transcriptions/accueil2.htm. 